\title{
Skyhook-PID Control Strategy to Improve Performance of a Pneumatic Active Suspension System
}

\author{
G. Priyandoko ${ }^{*}$, M. Mailah $^{2}$ \\ ${ }^{1}$ Department of Electrical Engineering, Faculty of Engineering, University of Widyagama, Malang, Indonesia \\ ${ }^{2}$ Faculty of Mechanical Engineering, Universiti Teknologi Malaysia, Malaysia \\ *Email: gigih@widyagama.ac.id
}

\begin{abstract}
The research applies a skyhook-PID control method for an active suspension system. The control strategy has three feedback control loops. They are the innermost loop for the force tracking of the pneumatic actuator, the intermediate loops applying skyhook strategy for the elimination of the disturbances, and the outermost loop using PID controller for the determination of the desired force. Some experiments were carried out on a physical test rig with a hardware-in-the-loops feature. The performance of the proposed control method was evaluated and benchmarked to examine the effectiveness of the system in suppressing the disturbance effect of the suspension system. It was found that the experimental results demonstrate the superiority of the active suspension system with Skyhook-PID scheme compared to the PID and passive suspension systems.
\end{abstract}

Keywords PID, Skyhook, Pneumatic, Active Suspension

Paper type Research paper

\section{INTRODUCTION}

A suspension system is one of the essential components of a car that carries a passenger. It is used to provide the absorption and isolation of undesirable vibration and load in the event the vehicle travels on a rough road, thereby, providing some comfort to the passenger in the vehicle [1]. Most vehicle suspension systems are typically made up of a spring and a shock absorber. When a car hits a bump or a hole, the spring is used to temporarily store the energy generated by the disturbance force and resist the motion that tends to change the car body height level. The shock absorber acts to quickly dissipate the energy stored in the spring and damp out the vibration. Without the shock absorber, the spring will cause the vehicle to vibrate continuously over a certain period after the tire passes over a bump or a hole.

An example can be seen in an automotive system in which the passenger/s of a car should ideally be isolated from vibration effects of the car's body when the car hits a bump or hole. In a conventional passive suspension system, the mass-spring-damper elements are generally fixed and are chosen based on the design requirements of the vehicles. The passive suspension was utilising springs and dampers to isolate the vibration of the vehicle body. In any vehicle suspension system, some performance parameters need to be optimised. In order to solve the problems, many researchers have studied numerous active vehicle suspension strategies both theoretically and experimentally [1]. Many researchers are proposed for complicated control strategy to eliminate disturbances. The results showed that such active suspension systems give relatively more satisfactory performance compared with the linear active suspension systems as reported in [2-5]. Intelligent control of the suspension system was also proposed using fuzzy logic [2-3] and neural network [4].

This paper aims to develop a hardware-in-the-loop (HILS) control strategy to minimise the sprung mass motion of a quarter car with the proposed control methods. The control strategy has three loops, first, the innermost control loop for force tracking control of the pneumatic actuator applying PI controller; and the outer positional control loop utilising a PID controller to generate the target force. Performance of the system is evaluated the ability to reduce the sprung mass acceleration, sprung mass displacement, suspension deflection and tire deflection in the presence of road disturbances.

\section{DESIGN OF THE CONTROL SYSTEM}

The complete proposed strategy called the skyhook-PID control strategy is shown schematically in Figure 1. The overall control system mostly comprises three feedback control loops to cater to some specific tasks. In this configuration, the inner loop controller must be able to track the optimum target force of the pneumatic actuator. Once the inner loop controller of the actuator can track well the target force with acceptable error, the actuator model and the inner loop controller are integrated with the outer loop of the active suspension control [6-8]. The typical transfer function of a PID controller is given as follows [9]. 


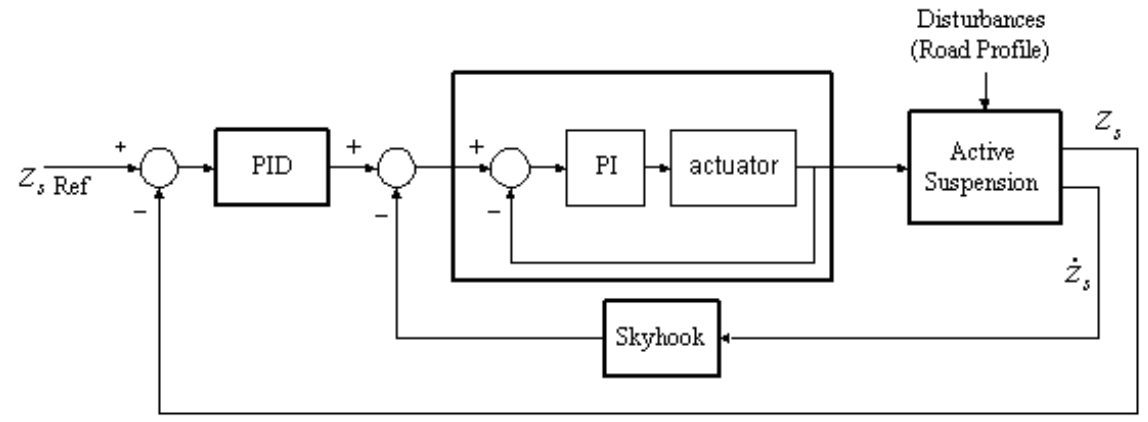

Fig. 1. The skyhook-PID control strategy

$$
P I D=K_{p}\left(e+\frac{1}{T_{i}} \int_{0}^{t} e d t+T_{d} \frac{d e}{d t}\right)
$$

where $\mathrm{Ti}=\mathrm{Kp} / \mathrm{Ki}, \mathrm{Td}=\mathrm{Kd} / \mathrm{Kp}$, and e is the error between the reference and the output system, Ti is the integral time, $\mathrm{Td}$ is derivative time, and $\mathrm{Kp}, \mathrm{Ki}$, and $\mathrm{Kd}$ are proportional, integral, and derivative gains, respectively.

In the intermediate loop, the skyhook method is applied. The idea of skyhook control has been in existence for more than three decades. The skyhook damper is known a 'fictitious' damper hooked up to the sky. It was first introduced by Crosby and Karnopp in 1974 [10], the skyhook control has been studied most often for vehicle suspension applications where a fictitious damper is inserted in between the sprung mass and the stationary sky as a way to suppress vibration of the sprung mass. The skyhook control is a simple concept and provides an excellent vibration suppression capability that reduces the resonant peak of the sprung mass. The implementation of the skyhook control requires information on the relative velocity of the sprung and unsprung mass as well as the absolute velocity of the sprung mass. However, the skyhook damper alone cannot reduce both resonant peaks at the same time [10].

$$
F_{s k y}=\left\{\begin{array}{cl}
-B_{s k y} \dot{z}_{s} & \text {, if } \dot{z}_{s}\left(\dot{z}_{s}-\dot{z}_{u}\right) \geq 0 \\
0 & \text {, if } \dot{z}_{s}\left(\dot{z}_{s}-\dot{z}_{u}\right)<0
\end{array}\right.
$$

where $B_{s k y}$ is a constant value of the skyhook often determined by a trial-and-error method.

After the innermost loop controller of the pneumatic actuator can track well the target forces using various input signals with acceptable error margin, the pneumatic actuator model and the inner loop controller are then integrated into the intermediate and outermost loops control of the active suspension system. The input of the outermost loop controller is body displacement (zs) error signal, whereas the output is the target force that must be tracked by the pneumatic actuator.

\section{RESULTS AND DISCUSSION}

The practical implementation of the skyhook-PID scheme is presented in Figure 2. A quarter-car suspension system is used with a pneumatic actuator as the main active element. HILS approach is used in conjunction with the Real-Time Workshop (RTW) feature in MATLAB/Simulink software. It is used to provide an effective procedure for developing and testing the performance of the physical suspension test rig in real-time. Interfacing between the hardware with the controller scheme through the data acquisition system (DAS). The actuator provides the force needed to eliminate the disturbances on the suspension test rig. By applying the HILS approach, some factors related to the actuator dynamics, the nonlinearity of the pneumatic system and time delay are assumed to be small and can be neglected in the modelling. Experimental studies investigate the benefits of active suspension using skyhookPID over a PID controller for a sinusoidal road input frequency $1.5 \mathrm{~Hz}$, amplitude $3.5 \mathrm{~cm}$. In this system, the innermost loop controller must be able to track the optimum target force of the pneumatic actuator. In order to produce the best results, the actuator must be able to track the desired force with acceptable accuracy. Some signals were tested to observe the adaptability of the controller against various types of signals. The experimental results are shown in Figure 3, which clearly shows that the actual trajectories are capable of tracking the desired ones. The signifies that the appropriate controller setting enables the actuator to operate satisfactorily.

In the intermediate loop, the Bsky is a constant value of the skyhook often determined by a trial-and-error method. In Figure 4 show that the $B_{s k y}=2900 \mathrm{Ns} / \mathrm{m}$ performs slightly better than $B_{s k y}=3000 \mathrm{Ns} / \mathrm{m}$ and significantly better than $B_{s k y}=1000 \mathrm{Ns} / \mathrm{m}$ and $B_{s k y}=2000 \mathrm{Ns} / \mathrm{m}$. The benefits and effectiveness of the pneumatic active suspension using the skyhook-PID controller over the other control scheme were verified through a rigorous experimental study. The results can be seen in Figure 5. The disturbance is applied sequentially, first to the PID 
controller and finally the skyhook-PID scheme. For the PID and skyhook-PID schemes, the first $10 \mathrm{~s}$ is when only the PID controller is operated. At the same time, for the next 10 seconds, the SPID mode is triggered using an automatic switch created in MATLAB/Simulink environment via the HILS configuration. From Figure 5, the amplitudes of sprung mass acceleration, the sprung mass displacement and the suspension deflection for active suspension based on skyhook-PID scheme show much better results compared with the PID controller counterpart.

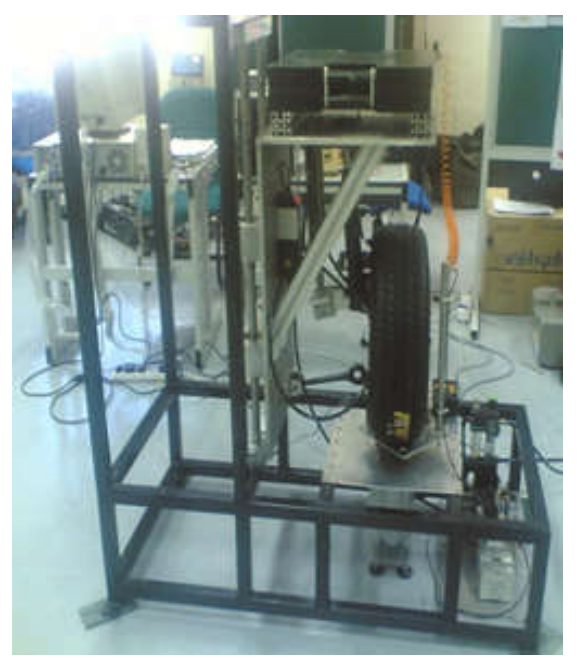

Fig. 2. The actual active suspension system.
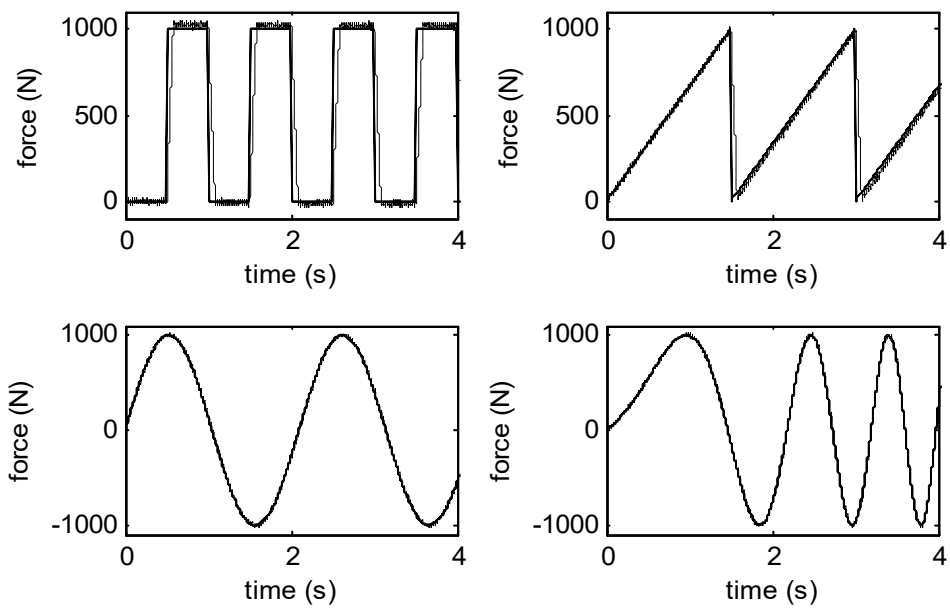

- actual -....- desired

Fig. 3. The force tracking control results.

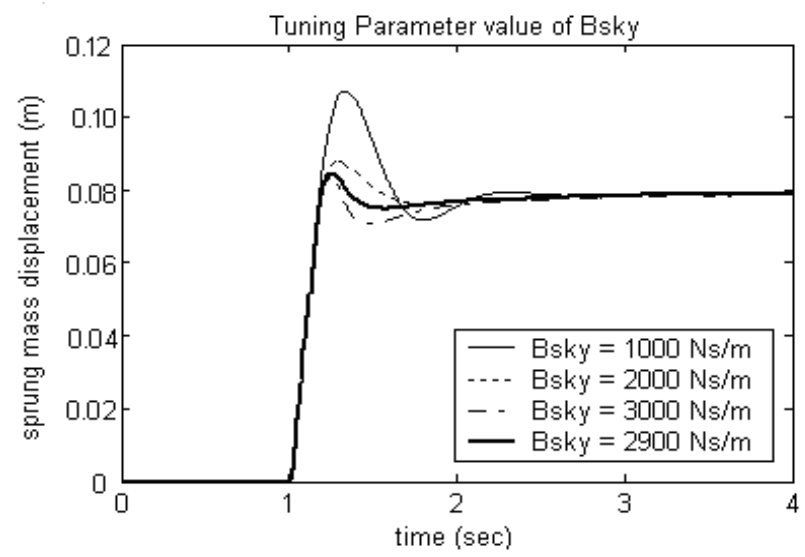

Fig. 4. Tuning $B_{s k y}$ for the skyhook method 

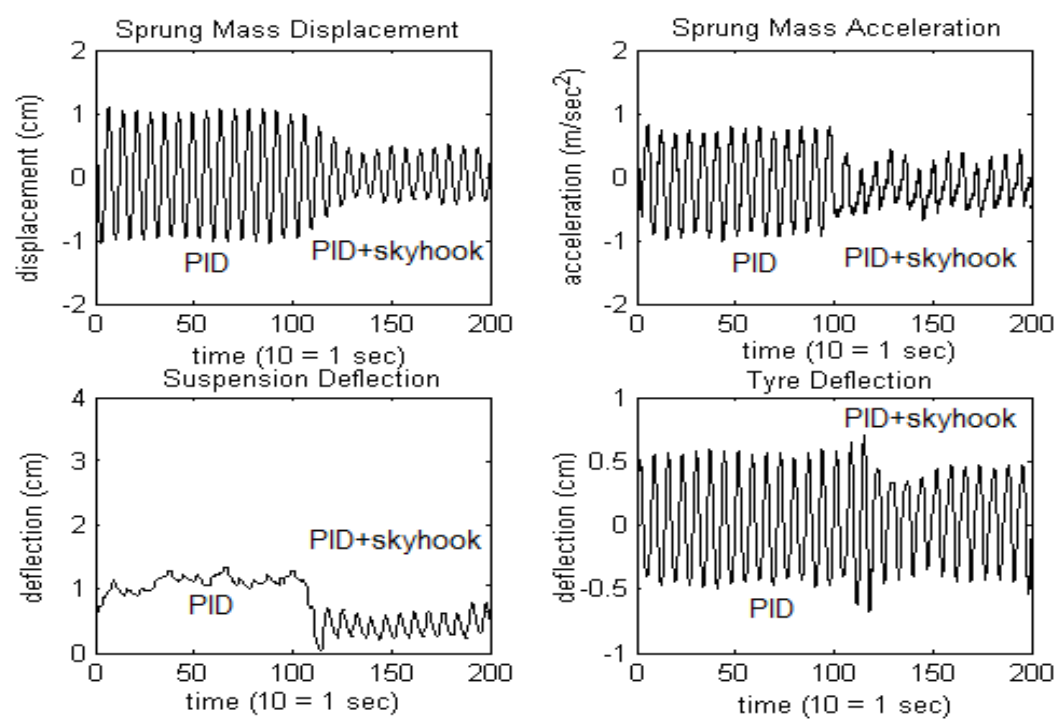

Fig.5. The actual active suspension system.

\section{CONCLUSION}

A control strategy called the skyhook-PID scheme for a quarter car active suspension system has been proposed, designed and implemented through a rigorous experimental study. It is a hybrid control scheme that integrates three main control loops to consolidate the overall system performance. The proposed system produced improved ride comfort (reduced peak values of vertical body acceleration and tyre deflection) performance in the wake of a disturbance in the form of a type road profile. The performance of the scheme in the time domain is generally better compared to the PID control schemes considered in the study. It can be deduced that the active suspension based on the skyhook-PID controller outperforms the PID controller in all selected performance criteria.

\section{REFERENCES}

[1] H. E. Tseng and D. Hrovat, "State of the Art Survey: Active and Semi-active Suspension Control," Vehicle System Dynamics, 53(7), pp.1034-1062, 2015.

[2] H. Li, X. Jing, H. K. Lam and P. Shi, "Fuzzy Sampled-data Control for Uncertain Vehicle Suspension Systems," IEEE Transactions on Cybernetics, 44(7), pp.1111-1126, 2014.

[3] J. Lin, R. J. Lian, C. N. Huang and W. T. Sie, "Enhanced Fuzzy Sliding Mode Controller for Active Suspension Systems," Mechatronics, Vol. 19, pp.1178-1190, 2009.

[4] Y. Zhu and S. Zhu, "Nonlinear Time-delay Suspension Adaptive Neural Network Active Control," In Abstract and Applied Analysis, Vol. 2014, Hindawi Publishing Corporation, 2014.

[5] G. Priyandoko, M. Mailah and H. Jamaluddin, "Vehicle Active Suspension System using Skyhook Adaptive Neuro Active Force Control," Mechanical Systems and Signal Processing, 23(3), pp.855-868, 2009.

[6] A. Alleyne and J. K. Hedrick, "Nonlinear Adaptive Control of Active Suspensions," IEEE Transactions on Control Systems Technology, 3(1), pp. 94-101, 1995.

[7] S. Chantranuwathana and H. Peng, "Force Tracking Control for Active Suspensions - Theory and Experiments," Proceedings of the 1999 IEEE International Conference on Control Applications, Vol. 1, pp. 442-447, 1999.

[8] Y. Zhang, and A. Alleyne, "A Practical and Effective Approach to Active Suspension Control," in Proceedings of the 6th International Symposium on Advanced Vehicle Control, Hiroshima, Japan, September - 2002.

[9] M. A. Johnson and M. H. Moradi, "PID Control: New Identification and Design Methods," SpringerVerlag, London, UK, 2005.

[10] C. Reginaldo and M. E. M. Meza, "Comparison of Two Fuzzy Skyhook Control Strategies Applied to an Active Suspension," World Academy of Science, Engineering and Technology, International Journal of Mathematical and Computational Sciences, 2, no. 9, 2015. 